Programa de Pós-Graduação em Engenharia de Produção - PPGEP

Laboratório de Qualidade de Vida - LaQVida

Universidade Tecnológica Federal do Paraná - UTFPR

Ponta Grossa - PR - Brasil

v. 01 , n. 02 , jul./dez. 2009 , p. $16-24$

DOI: $10.3895 / \mathrm{S} 2175-08582009000200003$

\section{REVISTA BRASILEIRA DE QUALIDADE DE VIDA}

\title{
Qualidade de vida de adolescentes da rede particular de ensino: comparação entre gêneros
}

\section{Quality of life of adolescents of the private net of education: comparison between genders}

\author{
Alex Pinheiro Gordia \\ Universidade Federal de Santa Catarina - UFSC - Florianópolis - Brasil \\ alexgordia@gmail.com \\ Teresa Maria Bianchini de Quadros \\ Universidade Federal de Santa Catarina - UFSC - Florianópolis - Brasil \\ tetemb@gmail.com \\ Wagner de Campos \\ Universidade Federal do Paraná - UFPR - Curitiba - Brasil \\ wagner@ufpr.br \\ Guanis de Barros Vilela Junior \\ Universidade Metodista de Piracicaba - UNIMEP - Piracicaba - Brasil \\ Universidade Estadual de Ponta Grossa - UEPG - Ponta Grossa - Brasil \\ Faculdade Integrada Metropolitana de Campinas - METROCAMP - Campinas - Brasil \\ guanis@gmail.com
}

\begin{abstract}
Resumo
O presente estudo teve como objetivo analisar a Qualidade de Vida (QV) de 49 adolescentes com idades entre 14 e 19 anos, estudantes de uma escola da rede particular de ensino da cidade de Curitiba, Paraná, bem como comparar a QV de rapazes e moças. O questionário WHOQOL-Bref foi utilizado para avaliar a QV dos adolescentes. Para a análise do WHOQOL-Bref utilizou-se os critérios propostos pela equipe australiana do WHOQOL com a QV classificada em uma escala de 0 a 100. Recorreu-se ao teste t de Student para avaliar possíveis diferenças na QV de adolescentes do sexo masculino e feminino. O nível de significância foi fixado em $p<0,05$. Os menores valores para os rapazes foram encontrados para o Domínio Meio Ambiente $(67,3)$, e para as moças o Domínio Psicológico apresentou-se como o mais preocupante, com o menor valor médio $(66,7)$. Não houve diferença significativa para a QV entre adolescentes do sexo masculino e feminino. Pode-se inferir que os Domínios mais preocupantes para a QV dos adolescentes investigados foram o Psicológico (para as moças) e o Meio Ambiente (para os rapazes e para a amostra total).
\end{abstract}

Palavras-chave: qualidade de vida; WHOQOL-Bref; adolescentes.

\section{Abstract}

This study aimed to analyze the Quality of Life (QL) in 49 adolescents aged 14 to 19 years, students at a private school from Curitiba, Paraná, and to compare the QL of males and females. The WHOQOL-Bref was used to evaluate the QL of adolescents. For the analysis of the WHOQOLBref was used the criteria proposed by WHOQOL Australian team with the QL classified into a scale from 0 to 100 . Student $t$ test was used to evaluate possible differences in QL of adolescent 
males and females. The level of significance was fixed at $p<0.05$. The lowest values for boys were found in the Domain Environment (67.3), and for girls the Psychological Domain was the most worrisome, with lowest average (66.7). There was no significant difference in QL between adolescent males and females. It can be inferred that the Domains most worrisome to the QL of adolescents surveyed were Psychological (for girls) and Environment (for boys and for the total sample).

Keywords: quality of life; WHOQOL-Bref; adolescents.

\section{Introdução}

A intensificação de pesquisas sobre o tema Qualidade de Vida (QV) é recente, entretanto há indícios de que o termo QV surgiu pela primeira vez na literatura médica na década de 30 (SEIDL; ZANNON, 2004). A QV é uma expressão que vem se tornando corriqueira no dia-a-dia das pessoas, mas que se reveste de grande complexidade, dada a subjetividade que representa para cada pessoa ou grupo social, podendo representar felicidade, harmonia, saúde, prosperidade, morar bem, ganhar salário digno, ter amor e família, poder conciliar lazer e trabalho, ter liberdade de expressão, ter segurança; podendo também pode significar todo esse conjunto de atributos e/ou benefícios (QUEIROZ; SÁ; ASSIS, 2004).

A QV de uma população depende de suas condições de existência e do seu acesso a certos bens e serviços econômicos e sociais, tais como, emprego e renda, educação básica, alimentação adequada, acesso a bons serviços de saúde, saneamento básico, habitação, transporte de boa qualidade, etc. (ADRIANO et al., 2000). Atualmente, o conceito QV tem sido utilizado em duas vertentes: (1) na linguagem cotidiana, por pessoas da população em geral, publicitários, agentes de marketing, políticos, profissionais de diversas áreas e gestores ligados às políticas públicas; (2) no contexto da pesquisa científica, em diferentes campos do saber, como Educação Física, Economia, Sociologia, Educação, Medicina, Enfermagem, Psicologia e demais especialidades da saúde (SEIDL; ZANON, 2004).

Pode-se definir a QV como a percepção do indivíduo sobre a sua posição na vida, no contexto da cultura e dos sistemas de valores nos quais ele vive, e em relação a seus objetivos, expectativas, padrões e preocupações (THE WHOQOL GROUP, 1995). Segundo Nahas (2003), a QV é a condição humana resultante de um conjunto de parâmetros individuais e sócio-ambientais, modificáveis ou não, que caracterizam as condições em que vive o ser humano. Os parâmetros sócio-ambientais relacionados à QV são: moradia, transporte, segurança, assistência médica, condições de trabalho, educação, opções de lazer e meio-ambiente; e os parâmetros individuais são: hereditariedade e estilo de vida.

A noção de QV transita em um campo semântico polissêmico: de um lado, está relacionada a modo, condições e estilo de vida; de outro, inclui as idéias de desenvolvimento sustentável e ecologia humana. E, por fim, relaciona-se ao campo da democracia, do desenvolvimento e dos direitos humanos e sociais. No que concerne à saúde, as noções se unem em uma resultante social da construção coletiva dos padrões de conforto e tolerância que determinada sociedade estabelece, como parâmetros para si (MINAYO; HARTZ; BUSS, 2000). Nota-se, através da conceituação da $\mathrm{QV}$, que se faz necessário a realização de diversos fatores para que se possa atingi-la, tornando-se assim, muito difícil a sua mensuração. As técnicas criadas para medir a QV, geralmente, não levam em conta o contexto cultural, social, de história de vida e do percurso dos indivíduos cuja QV pretendem mensurar (MINAYO, HARTZ; BUSS, 2000).

Tentando sintetizar a complexidade da noção de QV e de sua relatividade vis-à-vis as diferentes culturas e realidades sociais, diversos instrumentos têm sido construídos (MINAYO, HARTZ; BUSS, 2000). Os instrumentos mais relacionados à análise de condições gerais da QV enfatizam questões sobre domínios físicos (dor, fadiga, capacidades e limitações), psicológicos (percepção do estado de saúde, depressão, auto-estima, ansiedade e imagem corporal), relações sociais (apoio familiar e social, limitações impostas pela sociedade e as relações interpessoais), 
nível de independência (mobilidade, atividades cotidianas, capacidade para o trabalho) e noções sobre o bem-estar (corporal, emocional, saúde mental e vitalidade).

Um dos instrumentos mais utilizados atualmente para a avaliação da QV é um questionário desenvolvido pela Organização Mundial da Saúde. Este questionário, denominado WHOQOL-100 (por conter 100 questões), avalia seis domínios: psicológico, físico, nível de independência, relações sociais, ambiente e espiritualidade. Cada domínio é constituído por facetas que são avaliadas por quatro questões. Assim, o instrumento é composto por 24 facetas específicas e uma faceta geral que inclui questões de avaliação global de QV.

Porém, em busca de um instrumento menos complexo e de rápida aplicação, desenvolveu-se o WHOQOL-Bref, um questionário (com a mesma essência do WHOQOL-100) contendo 26 questões circunscritas a quatro domínios: físico, psicológico, relações sociais e meio ambiente. O critério de seleção das questões para compor a versão abreviada foi tanto psicométrica como conceitual. Este instrumento está recebendo alta aceitação e foi testado e validado em diversos países, inclusive no Brasil (FLECK et al., 2000; MORENO et al., 2006). A aplicação do WHOQOL-Bref é ampla e inclui não somente a prática clínica individual, mas também a avaliação da efetividade de tratamentos e do funcionamento de serviços de saúde, podendo ser um importante guia para promoção de políticas públicas.

Recentemente está aumentando o interesse dos diversos profissionais da saúde na QV de crianças e adolescentes, tendo em vista que esta é uma fase chave para intervenções e modificações nos hábitos de vida. Entretanto, a maioria dos estudos e instrumentos focaliza somente crianças e adolescentes que possuem doenças crônicas, e desta forma, os aspectos relacionados à QV de crianças e adolescentes saudáveis ainda são pouco estudados. Além disso, estudos que visem comparar a QV de adolescentes do sexo masculino e feminino são escassos. Neste sentido, esta investigação objetivou analisar a QV de adolescentes estudantes de uma escola da rede particular de ensino da cidade de Curitiba, Paraná, bem como comparar a QV de rapazes e moças.

\section{Métodos}

População e Amostra

Este estudo foi realizado com estudantes de um Colégio Particular da cidade de Curitiba, Paraná. O Colégio em questão possuía 57 alunos matriculados no Ensino Médio no ano de 2006, e a amostra da presente investigação foi composta por 49 adolescentes com idades entre 14 e 19 anos, estudantes da $1^{\mathrm{a}}, 2^{\mathrm{a}}$ e $3^{\mathrm{a}}$ série do Ensino Médio. As informações descritivas sobre a amostra estudada podem ser observadas na Tabela 1.

\section{Esclarecimentos e Termo de Consentimento}

Em uma primeira visita ao Colégio houve um prévio esclarecimento aos participantes sobre o desenvolvimento e objetivos da pesquisa, bem como entrega do termo de consentimento livre e esclarecido. Em uma segunda visita, os adolescentes que manifestaram interesse em participar da pesquisa entregaram o termo de consentimento devidamente assinado por pais e/ou responsáveis quando menores de 18 anos de idade. Os adolescentes com 18 anos de idade ou mais que participaram do estudo assinaram seus respectivos termos de consentimento.

\section{Critérios e Inclusão/Exclusão}

O limite da faixa etária para participar do estudo foi estabelecido através do critério da Organização Mundial da Saúde (OMS), condiderando a adolescência como o período da vida que se estende dos 10 aos 20 anos de idade (WHO, 1977). Desta amostra foi excluído um estudante do sexo masculino que apresentou idade superior a 20 anos. Participaram do estudo apenas adolescentes que estavam presentes no dia da coleta de dados, desta forma sete estudantes (quatro adolescentes do sexo masculino e três do sexo feminino) que não compareceram ao Colégio neste dia não participaram da investigação, totalizando a amostra final em 49 adolescentes $(86,0 \%$ da população), 34 do sexo masculino e 15 do sexo feminino. 
Instrumento

Para avaliação da QV utilizou-se o questionário WHOQOL-Bref, desenvolvido pelo grupo de estudos sobre QV da OMS em 1995. A constatação de que não havia nenhum instrumento que avaliasse QV dentro de uma perspectiva transcultural motivou a OMS a desenvolver um instrumento com estas características (FLECK et al., 1999). Nesta perspectiva, a OMS desenvolveu o WHOQOL-100, um instrumento que baseia-se nos pressupostos de que a QV é uma construção subjetiva (percepção do indivíduo em questão), multidimensional e composta por elementos positivos (por exemplo, mobilidade) e negativos (dor) (MINAYO; HARTZ; BUSS, 2000).

No entanto, a necessidade de um instrumento mais curto para uso em extensos estudos epidemiológicos, que demandasse pouco tempo para o preenchimento e que preservasse características psicométricas satisfatórias, fez com que o Grupo de QV da OMS desenvolvesse uma versão abreviada do WHOQOL-100, o WHOQOL-Bref. Fleck et al. (2000) mostraram que a versão abreviada em português no Brasil do WHOQOL-Bref apresenta características satisfatórias de consistência interna, validade discriminante, validade de critério, validade concorrente e fidedignidade teste-reteste.

O instrumento WHOQOL-Bref é composto por 26 questões e considera os últimos quinze dias vividos pelos respondentes. Duas questões referem-se a percepção individual a respeito da QV e as demais 24 estão subdivididas em quatro domínios, e representam cada uma das 24 facetas que compõem o instrumento original (WHOQOL-100), tais como: Domínio I - Físico, com ênfase nas seguintes facetas: dor e desconforto, energia e fadiga, sono e repouso, mobilidade, atividades da vida cotidiana, dependência de medicação ou de tratamentos e capacidade de trabalho; Domínio II Psicológico, focalizando as seguintes facetas: sentimentos positivos, pensar, aprender, memória e concentração, auto-estima, imagem corporal e aparência, sentimentos negativos, espiritualidade, religião e crenças pessoais; Domínio III - Relações sociais, abordando as facetas: relações pessoais, suporte (apoio) social, atividade sexual; Domínio IV - Meio ambiente, com as facetas: segurança física e proteção, ambiente no lar, recursos financeiros, cuidados de saúde e sociais: disponibilidade e qualidade, oportunidade de adquirir novas informações e habilidades, participação e oportunidades de recreação/lazer, e ambiente físico (poluição, ruído, trânsito, clima e transporte) (FLECK et al., 2000).

Ainda que o WHOQOL-Bref não tenha sido validado para adolescentes no Brasil, Izutsu et al. (2005) demonstraram que este instrumento possui um conteúdo válido e propriedades psicométricas aceitáveis para mensurar a QV de adolescentes.

\section{Coleta de Dados}

A coleta dos dados foi realizada no mês de novembro de 2006. Um único pesquisador foi responsável pela aplicação do WHOQOL-Bref, com o intuito de minimizar as possibilidades de equívoco durante a mensuração da QV. O questionário foi administrado através da aplicação coordenada, com o aplicador lendo a pergunta para os alunos, com o propósito de facilitar o entendimento dos respondentes em relação ao objetivo de cada questão.

\section{Análise do WHOQOL-Bref}

Para a análise do questionário sobre QV utilizou-se os critérios propostos pela equipe australiana do WHOQOL, pela clareza e praticidade com que são demonstradas todas as fases de análise e interpretação dos resultados obtidos. A sintaxe do software estatístico SPSS versão 11,5 (SPSS Inc.) foi utilizada para análise da QV, sendo classificada em uma escala de 0 a 100 . A consistência interna das respostas foi analisada através do coeficiente de fidedignidade de Cronbach.

\section{Tratamento Estatístico}

Em um primeiro momento, recorreu-se a análise descritiva através dos valores da média e desvio padrão dos indicadores utilizados para verificar o comportamento da amostra em relação à 
QV. Em um segundo momento, recorreu-se ao teste de Kolmogorov-Smirnov para avaliar a normalidade dos dados. Tendo em vista que os dados apresentaram distribuição normal, em um terceiro momento, utilizou-se o teste $\mathrm{t}$ de Student para amostras independentes, visando avaliar possíveis diferenças na QV de adolescentes do sexo masculino e feminino. O nível de significância foi fixado em $\mathrm{p}<0,05$.

Tabela 1 - Informações descritivas dos participantes referentes à idade (média \pm desvio padrão) e número de indivíduos por sexo

\begin{tabular}{|c|c|c|c|}
\hline \multirow{2}{*}{ Adolescentes estudados } & \multicolumn{2}{|c|}{ Participantes (n) } & \multirow{2}{*}{ Idade (média $\pm D P$ ) } \\
\hline & Freqüência absoluta & Freqüência relativa & \\
\hline Masculino & 35 & $71,4 \%$ & $16,00 \pm 1,50$ \\
\hline Feminino & 14 & $28,6 \%$ & $15,29 \pm 0,91$ \\
\hline Total & 49 & $100 \%$ & $15,80 \pm 1,38$ \\
\hline
\end{tabular}

\section{Resultados}

Com base na análise do WHOQOL-Bref obtiveram-se os resultados referentes à QV global e subdividida em: Domínio I - Físico, Domínio II - Psicológico, Domínio III - Relações Sociais, e Domínio IV - Meio Ambiente (Tabela 2). A consistência interna das respostas do WHOQOL-Bref, obtida através do coeficiente de fidedignidade de Cronbach foi de $\alpha=0,85$.

Os menores valores percentuais para os rapazes foram encontrados para o Domínio IV Meio Ambiente (67,3), sendo este o aspecto mais vulnerável e preocupante para a QV deste grupo. Para as moças o Domínio II - Psicológico apresentou-se como o mais preocupante, com o menor valor médio $(66,7)$. Os melhores resultados, tanto para rapazes quanto para moças, foram para o Domínio III - Relações Sociais (75,0 e 79,2, respectivamente), indicando que este domínio é o principal contribuinte para elevação da QV global. Quando a amostra não foi subdividida por sexo, pode-se observar que o Domínio Meio Ambiente apresentou o menor valor percentual médio.

Tabela 2 - Domínios do WHOQOL-Bref e QV global dos adolescentes por sexo e amostra total com valores percentuais expressos em média e desvio padrão

\begin{tabular}{lccc}
\hline Qualidade de Vida & Masculino & Feminino & Amostra total \\
\hline Domínio I - Físico & $73,7 \pm 11,7$ & $71,9 \pm 16,3$ & $73,2 \pm 13,0$ \\
Domínio II - Psicológico & $71,7 \pm 12,7$ & $66,7 \pm 19,7$ & $70,2 \pm 14,9$ \\
Domínio III - Relações Sociais & $75,0 \pm 17,6$ & $79,2 \pm 15,9$ & $76,2 \pm 17,1$ \\
Domínio IV - Meio Ambiente & $67,3 \pm 12,3$ & $71,0 \pm 13,6$ & $68,4 \pm 12,6$ \\
QV Global & $71,9 \pm 10,5$ & $72,2 \pm 13,4$ & $72,0 \pm 11,2$ \\
\hline
\end{tabular}

Através dos resultados do teste $t$ de Student para amostras independentes pode-se observar que não houve diferença significativa para a QV global entre adolescentes do sexo masculino e feminino $(t=0,074 ; p=0,941)$, bem como não foram identificadas disparidades entre os gêneros para o Domínio Físico ( $t=0,423 ; p=0,674)$, Psicológico $(t=1,059 ; p=0,295)$, Relações Sociais $(t=0,769$; $\mathrm{p}=0,446)$ e Meio Ambiente $(\mathrm{t}=0,915 ; \mathrm{p}=0,365)$.

\section{Discussão}

O desenvolvimento da QV pode resultar em mudanças nas práticas assistenciais e na consolidação de novos paradigmas do processo saúde-doença, o que pode ser de grande valia para a superação de modelos de atendimento eminentemente biomédicos, que negligenciam aspectos socioeconômicos, psicológicos e culturais importantes nas ações de promoção, prevenção, tratamento e reabilitação em saúde. Tendo em vista que a QV é um construto eminentemente interdisciplinar, a contribuição de diferentes áreas do conhecimento pode ser de fato valiosa e mesmo indispensável (SEIDL; ZANNON, 2004).

Nas últimas décadas, o interesse crescente da comunidade científica e de todo o setor de saúde em questões de QV levou a um desenvolvimento marcante desse construto (CAMPOLINA; 
CICONELLI, 2006). No entanto, quando se fala em QV é preciso referir-se ao aspecto multidimensional de um conceito que envolve perspectivas interdisciplinar e intersetorial em sua interação com o entorno social e ambiental. $\mathrm{O}$ desafio central de investigações referentes à análise da QV de diversas populações é o de produzir análises contextualizadas e transformadoras da realidade e que fujam do paradigma médico-assistencialista, ampliando as alternativas de promoção e prevenção da saúde (UCHOA; ROZEMBERG; PORTO, 2002).

O questionário WHOQOL-Bref, desenvolvido pela OMS, mostrou-se um instrumento eficiente para a avaliação da QV dos indivíduos estudados. A versão abreviada do WHOQOL-100 preserva a abrangência do construto " $\mathrm{QV}$ " incluindo itens não só referentes a aspectos físicos e psicológicos, mas também relativos ao meio ambiente e relações sociais. Kao et al. (2005) objetivaram examinar a eficácia do WHOQOL-Bref na predição de mortalidade no período de 2 anos em 689 indivíduos do sexo masculino com idade superior a 65 anos. Os autores inferiram que o WHOQOL-Bref foi capaz de predizer a mortalidade dos indivíduos estudados, evidenciando a importância da utilização deste instrumento em programas de saúde pública e revelando a necessidade da realização de pesquisas epidemiológicas sobre a QV.

Os resultados observados através da análise do WHOQOL-Bref indicam que, de uma forma geral, a QV dos adolescentes investigados pode ser considerada satisfatória, levando-se em consideração que a maioria das médias dos Domínios foi superior a 70. Embora existam diferenças metodológicas, principalmente em relação às características amostrais, outros estudos que utilizam o WHOQOL-Bref têm encontrado resultados relativamente inferiores aos observados no presente estudo para QV (SIVIERO, 2003; GORDIA; QUADROS; VILELA JÚNIOR, 2006; GORDIA et al., 2007; GORDIA et al., 2009).

Com base nos resultados do teste $t$ de Student para amostras independentes pode-se verificar que a QV dos rapazes e moças estudados não apresentou diferenças, tanto em relação à QV global, quanto para os Domínios Físico, Psicológico, Relações Sociais e Meio Ambiente. Poucas pesquisas vêm sendo desenvolvidas com o objetivo de comparar a QV de adolescentes do sexo masculino e feminino, desta forma o confronto com resultados de outros estudos fica prejudicado. Vilela Junior et al. (2007) avaliaram diferenças na QV de homens e mulheres acadêmicos do primeiro e segundo ano do curso de Educação Física e Esporte de uma Faculdade particular de Campinas-SP. Os resultados demonstram que os indivíduos do sexo masculino apresentaram resultados significativamente melhores do que as mulheres em todos os domínios do WHOQOL-Bref, bem como para a QV global. Levando-se em consideração as diferenças observadas entre o presente estudo e a pesquisa de Vilela Junior et al. (2007), bem como a falta de estudos que avaliam a QV em adolescentes, sugere-se que mais estudos sejam desenvolvidos buscando analisar diferenças na QV de indivíduos do sexo masculino e feminino, principalmente na população adolescente, para que futuros programas de intervenção sejam eficazes.

O questionário WHOQOL-Bref possibilita tanto o fracionamento da QV em Domínios (Físico, Psicológico, Relações Sociais e Meio Ambiente) quanto a quantificação da QV global. Este diferencial é bastante interessante, tendo em vista que pode-se identificar quais aspectos da vida do indivíduo analisado são mais preocupantes e requerem intervenções. Os Domínios mais preocupantes para os adolescentes investigados foram o Psicológico (para as moças) e o Meio Ambiente (para os rapazes e para a amostra total).

Os baixos valores para o Domínio Psicológico entre as moças estão relacionados a problemas referentes às seguintes facetas: sentimentos positivos e negativos, pensar, aprender, memória e concentração, auto-estima, imagem corporal e aparência, espiritualidade, religião e crenças pessoais. Desta forma, acredita-se que moças tenham uma visão diferenciada e mais crítica em relação à sua QV no que diz respeito a questões como imagem corporal e auto-estima, entretanto faz-se necessário a avaliação de outras variáveis, através de instrumentos específicos, para que os baixos valores observados para o Domínio Psicológico para adolescentes do sexo feminino possam ser mais bem explicados.

A maioria dos estudos que utilizaram o WHOQOL no Brasil demonstraram que o Domínio Meio Ambiente é a pior faceta da QV da população brasileira. Gordia, Quadros e Vilela Júnior 
(2006), na analise da QV de indivíduos do Exército Brasileiro, obtiveram os menores valores para este domínio, sendo, portanto, o ponto mais vulnerável na QV da população estudada. O mesmo pode ser observado em outras populações; Gordia et al. (2007) avaliaram a QV de mulheres idosas da cidade de Fortaleza, CE, e os achados indicaram índices preocupantes para o aspecto ambiental da QV. Em pesquisa desenvolvida por Siviero (2003), com 33 pacientes pós-infarto com idade igual ou inferior a 59 anos, também se obteve resultados semelhantes aos do presente estudo, com o domínio ambiental apresentando valores inferiores aos outros domínios.

Esta tendência de baixos valores para o domínio ambiental é preocupante, pois está diretamente vinculada a falta de investimento em políticas públicas. Este domínio trata da segurança física e proteção, ambiente no lar, recursos financeiros, cuidados de saúde e sociais: disponibilidade e qualidade, oportunidade de adquirir novas informações e habilidades, participação e oportunidades de recreação/lazer, ambiente físico: poluição, ruído, trânsito, clima e transporte. Como se pode observar, neste domínio existem fatores que não podem ser controlados individualmente, sendo necessária a intervenção de órgãos governamentais.

Recentemente, alguns municípios brasileiros (por exemplo, Curitiba-PR, Chopinzinho-PR, Vinhedo-SP), preocupados com a melhoria das condições de vida da população, estão implementando programas de intervenção para melhoria de diversos fatores, tais como: saneamento básico, educação, assistência médica, ambientes de trabalho e lazer saudável (VILARTA, 2004). Entretanto, o comprometimento dos governantes com a melhoria das condições de vida dos brasileiros ainda é muito reduzido, e este descaso está explícito nos resultados obtidos no presente estudo.

Evidentemente torna-se limitado avaliar exclusivamente por meio de quantificações um conceito intrinsecamente marcado pela subjetividade, como o construto QV. É preciso ter em mente que os indicadores e índices medem sempre "aspectos" da QV, tendo méritos e limitações uns em relação aos outros (TAMAKI, 2000). Neste sentido, os resultados observados no presente estudo precisam ser analisados com cautela, tendo em vista que a medida "objetiva" da QV foi mensurada de forma subjetiva através de questionário. No entanto, é importante ressaltar que o instrumento utilizado (WHOQOL-Bref) tem se apresentado como uma possibilidade de análise da QV prática e confiável, em adição, o WHOQOL-Bref é recomendado pela OMS para avaliação da QV (THE WHOQOL GROUP, 1998).

\section{Conclusão}

Com base nos achados do presente estudo pode-se inferir que não há diferença entre os gêneros para QV de estudantes de uma escola da rede particular de ensino da cidade de Curitiba, Paraná. Em adição, os Domínios mais preocupantes para a QV dos adolescentes investigados foram o Psicológico (para as moças) e o Meio Ambiente (para os rapazes e para a amostra total), indicando que estratégias de intervenção devem ser direcionadas visando alterações em facetas referentes a questões psicológicas e ambientais da população estudada.

\section{Referências}

ADRIANO, J. R.; WERNECK, G. A. F.; SANTOS, M. A.; SOUZA, R. C. A construção de cidades saudáveis: uma estratégia viável para a melhoria da qualidade de vida? Ciência e Saúde Coletiva, v. 5, n. 1, p. 53-62, 2000. Disponível em: 〈http://www.scielo.br/pdf/csc/v5n1/7079.pdf〉. Acesso em: 23 jun. 2007.

CAMPOLINA, A. G.; CICONELLI, R. M. Qualidade de vida e medidas de utilidade: parâmetros clínicos para as tomadas de decisão em saúde. Revista Panamericana de Salud Pública, v. 19, n. 2, p. 128-136, 2006. Disponível em: <http://www.ppge.ufrgs.br/ats/disciplinas/10/campolinaiciconelli-2006.pdf>. Acesso em: 14 set. 2007. 
FLECK, M. P. A; LOUZADA, S.; XAVIER, M.; CHACHAMOVICH, E.; VIEIRA, G.; SANTOS, L.; PINZON, V. Aplicação da versão em português do instrumento de avaliação da qualidade de vida da Organização Mundial da Saúde (WHOQOL-100). Revista de Saúde Pública, v. 33, n. 2, p. 198-205, 1999. Disponível em: <http://www.scielo.br/scielo.php?script=sci_arttext\&pid=S0034$89101999000200012 \& \operatorname{lng}=$ pt\&nrm=iso\&tlng=pt $>$. Acesso em: 05 ago. 2005.

FLECK, M. P. A.; LOUZADA, S.; XAVIER, M.; CHACHAMOVICH, E.; VIEIRA, G.; SANTOS, L.; PINZON, V. Aplicação da versão em português do instrumento abreviado de avaliação da qualidade de vida "WHOQOL-bref". Revista de Saúde Pública, v. 34, n. 2, p. 178-83, 2000. Disponível em: <http://www.scielo.br/pdf/rsp/v34n2/1954.pdf>. Acesso em: 05 ago. 2005.

GORDIA, A. P.; QUADROS, T. M. B.; VILELA JÚNIOR, G. B. Quality of life and physical fitness of individuals in the brazilian army. The FIEP bulletin, v. 76, p. 82-85, 2006.

GORDIA, A. P.; QUADROS, T. M. B.; VILELA JÚNIOR, G. B.; SOUZA, E. A.; CABRAL, C.; MORAIS, T. B.; QUADROS JÚNIOR, P. K.; CAMPOS, W. Comparação da qualidade de vida de mulheres idosas praticantes e não praticantes de exercício físico. Lecturas: Educación Física y Deportes, v. 11, n. 106, 2007. Disponível em: <http://www.efdeportes.com/efd106/mulheresidosas-praticantes-de-exercicio-fisico.htm>. Acesso em: 26 nov. 2007.

GORDIA, A. P.; QUADROS, T. M. B.; PETROSKI, E. L.; CAMPOS, W. Domínio físico da qualidade de vida entre adolescentes: associação com atividade física e sexo. Revista de Salud Pública, v. 11, n. 1, p. 50-61, 2009. Disponível em: <http://www.scielosp.org/pdf/rsap/v11n1/v11n1a06.pdf>. Acesso em: 22 jun. 2009.

IZUTSU, T.; TSUTSUMI, A.; ISLAM, A.; MATSUO, Y.; YAMADA, H. S.; KURITA, H.; WAKAI, S. Validity and reliability of the Bangla version of WHOQOLBREF on an adolescent population in Bangladesh. Quality of Life Research, n. 40, p. 1783-1789, 2005. Disponível em: <http://www.springerlink.com/content/j1n3554823307556/>. Acesso em: 08 fev. 2006.

KAO, S.; LAI, K.; LIN, H.; LEE, H.; WEN, H. WHOQOL-Bref as predictors of mortality: A twoyear follow-up study at veteran homes. Quality of Life Research, n. 14, p. 1443-1454, 2005. Disponível em: <http://www.springerlink.com/content/k14h5636578qkk63/>. Acesso em: 24 mar. 2006.

MINAYO, M. C. S; HARTZ, Z. M. A.; BUSS, P. M. Qualidade de vida e saúde: um debate necessário. Ciência e Saúde Coletiva, v. 5, n. 1, p. 7-18, 2000. Disponível em: <http://www.scielo.br/pdf/csc/v5n1/7075.pdf>. Acesso em: 25 jul. 2005.

MORENO, A. B.; FAERSTEIN, E.; WERNECK, G. L.; LOPES, C. S.; CHOR, D. Propriedades psicométricas do Instrumento Abreviado de Avaliação de Qualidade de Vida da Organização Mundial da Saúde no Estudo Pró-Saúde. Cadernos de Saúde Pública, v. 22, n. 12, p. 2585-2597, 2006. Disponível em: <http://www.scielo.br/pdf/csp/v22n12/08.pdf>. Acesso em: 03 mai. 2007.

NAHAS, M. V. Atividade Física, Saúde e Qualidade de Vida: conceitos e sugestões para um estilo de vida ativo. 3 ed. Londrina: Midiograf, 2003. 
QUEIROZ, C. M. B.; SÁ, E. N. C.; ASSIS, M. M. A. Qualidade de vida e políticas públicas no município de Feira de Santana. Ciência e Saúde Coletiva, v. 9, n. 2, p. 411-421, 2004. Disponível em: <http://www.scielo.br/pdf/csc/v9n2/20395.pdf>. Acesso em: 17 nov. 2006.

SEIDL, E. M. F.; ZANNON, C. M. L. C. Qualidade de vida e saúde: aspectos conceituais e metodológicos. Cadernos de Saúde Pública, v. 20, n. 2, p. 580-88, 2004. Disponível em: <http://www.scielo.br/pdf/csp/v20n2/27.pdf>. Acesso em: 07 set. 2005.

SIVIERO, L. M. P. S. Saúde mental e qualidade de vida de infartados. 2003. Tese (Doutorado em Enfermagem) - Escola de Enfermagem de Ribeirão Preto, Universidade de São Paulo, Ribeirão Preto, 2003.

TAMAKI, E. M. Debate: qualidade de vida individual ou coletiva? Ciência e Saúde Coletiva, v. 5, n. 1, p. 19-31, 2000. Disponível em: <http://www.scielo.br/pdf/csc/v5n1/7076.pdf>. Acesso em: 12 abr. 2005.

THE WHOQOL GROUP. The World Health Organization quality of life assessment (WHOQOL): position paper from the World Health Organization. Social Science and Medicine, v. 41, p. 14031409 , 1995.

Disponível

em: <http://www.sciencedirect.com/science?_ob=ArticleURL\&_udi=B6VBF-3YS8D3J-

S\&_user $=10 \& \_r d o c=1 \& \_f m t=\& \_$orig $=$search \&_sort $=$d \&_docanchor $=\&$ view $=$ c \&_acct $=$ C00005022 $1 \&$ _version=1\&_urlVersion=0\&_userid=10\&md5=f20e9a $4 \mathrm{c} 27 \mathrm{dc} 7$ eeebcbb0fd55e $4 \mathrm{f} 6 \mathrm{fc} 3>$. Acesso em: 04 mai. 2005.

THE WHOQOL GROUP. The World Health Organization quality of life assessment (WHOQOL): development and general psychometric properties. Social Science and Medicine, v. 46, p. 15691585 , 1998.

Disponível

em: $<$ http://www.sciencedirect.com/science?_ob=ArticleURL\&_udi=B6VBF-3T3RW5S-

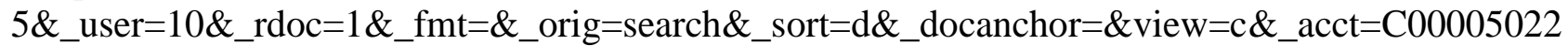
$1 \&$ \&ersion $=1 \&$ \&urlVersion $=0 \&$ \&userid $=10 \& \mathrm{md} 5=2928 \mathrm{a} 265906 \mathrm{c} 6152 \mathrm{adb} 1 \mathrm{c} 7 \mathrm{~b} 67 \mathrm{c} 426423>$.

Acesso em: 04 mai. 2005.

UCHOA, E.; ROZEMBERG, B.; PORTO, M. F. S. Entre a fragmentação e a integração: saúde e qualidade de vida de grupos populacionais específicos. Informe Epidemiológico do SUS, v. 11, n. 3, p. 115-128, 2002. Disponível em: 〈http://scielo.iec.pa.gov.br/pdf/iesus/v11n3/v11n3a02.pdf〉. Acesso em: 19 set. 2006.

VILARTA, R. (Org.). Qualidade de vida e políticas públicas: saúde, lazer e atividade física. Campinas: IPES Editorial, 2004.

VILELA JUNIOR, G. B.; GORDIA, A. P.; QUADROS, T. M. B.; CAMPOS, W. Relation between Quality of Life and alcohol consumption for students attending the Physical Education \& Sports Course. The FIEP Bulletin, v. 77, p. 231-235, 2007.

WORLD HEALTH ORGANIZATION. Expert Committee on Health Needs of Adolescent's. Geneva: World Health Organization; (Technical Report Series, 609), 1977. 